\title{
Introduction to the Commentaries
}

\author{
Misha E. Kilmer and Dianne P. O'Leary
}

In research spanning over 40 years, G.W. (Pete) Stewart has made foundational contributions to numerical linear algebra.

A major theme in this research is understanding the effects of small perturbations in the matrix on key quantities derived from it: its eigenvalues and eigenvectors, its invariant subspaces, and solutions to linear systems or least squares problems involving the matrix.

A second major theme is the development of efficient matrix algorithms. His insights range from the clever (e.g., economical storage of rotation matrices) to the elegant (the QZ algorithm for solving the generalized eigenproblem and the rank-revealing QR decomposition), and they are grounded in stable matrix decompositions and hands-on computational experience.

The following seven chapters of this commentary outline some of Stewart's important contributions to these two areas.

\section{Notation}

Throughout the commentaries, matrices and vectors will be indicated with boldface type, and all vectors are column vectors. Transpose is indicated by a superscript "T," and complex conjugate transpose by a superscript "H."

A specific norm will be identified by a subscript (e.g., 1, 2, F), while a norm without a subscript indicates any of a class of norms.

References to [GWS...] refer to the list of Stewart's publications in Sect. 2.1. 